\title{
Hypoxia-inducible adrenomedullin ameliorates the epithelial-to-mesenchymal transition in human proximal tubular epithelial cells
}

\author{
TIECHUI ZHU, JUN YANG, XIANGDONG LIU, LIANYUN ZHANG, JIE ZHANG, \\ YONGTAO WANG, HAIJUN MA and ZHENHUI REN \\ Department of Nephrology, First Affiliated Hospital of Xinxiang Medical University, \\ Xinxiang, Henan 453100, P.R. China
}

Received February 1, 2014; Accepted August 19, 2014

DOI: $10.3892 / \mathrm{mmr} .2015 .3189$

\begin{abstract}
Renal tubular epithelial cells can enter the epithelial-to-mesenchymal transition (EMT) in response to chronic hypoxia. EMT is a process which involves the phenotypic conversion of epithelial cells, that is believed to have an important role in renal fibrosis. However, the underlying mechanisms of the involvement of EMT in renal fibrosis remain to be elucidated. Adrenomedullin (AMD) has been implicated in renal fibrosis and is induced by hypoxia. The aims of the present study were to determine whether ADM signaling was active in human proximal tubular epithelial cells cultured under hypoxic conditions, and to observe the activity of ADM during EMT. The expression levels of ADM were significantly increased, in a time-dependent manner, in HK-2 and HKC human proximal tubular epithelial cells, cultured under hypoxic conditions. Overexpression of exogenous ADM was accompanied by increased expression levels of the epithelial markers E-cadherin and tight junction protein-1, and decreased expression levels of the mesenchymal markers vimentin and $\alpha$-smooth muscle actin, during hypoxia. Knock-down of ADM expression by small hairpin RNA, or co-administration of an ADM peptide inhibitor, in HK-2 cells significantly exacerbated hypoxia-induced EMT, as compared to the lack of effect observed in the untransfected controls. ADM was shown to suppress EMT by inhibiting the activation of extracellular signal-regulated kinase (ERK), and this effect was prevented by the ERK activator apigenin. The results of the present study suggest that ADM has an important role in promoting EMT in hypoxic human proximal tubular epithelial
\end{abstract}

Correspondence to: Dr Zhenhui Ren, Department of Nephrology, First Affiliated Hospital of Xinxiang Medical University, 88 Jiankang Road, Xinxiang, Henan 453100, P.R. China E-mail: zhenhui_Ren110@126.com

Key words: adrenomedullin, epithelial to mesenchymal transition, RNA interference, renal fibrosis, tubular epithelial cells cells. ADM may therefore represent a novel therapeutic target in the treatment of injured kidneys.

\section{Introduction}

End-stage renal disease (ESRD) is the terminal stage of irreversible chronic kidney disease (CKD), leading to a devastating condition that necessitates patient dependency on life-long treatments including dialysis, or renal transplantation $(1,2)$. The number of patients with CKD has been reported to be increasing at an annual rate of $\sim 7 \%$, and the human and economic impacts of ESRD to family members, the medical community and society is vast $(3,4)$. The majority of patients with CKD are diagnosed prior to reaching end-stage renal failure; however, there are no effective treatment options that can effectively halt the progressive decline in renal function. An improved understanding of the pathophysiology of CKD may lead to the development of novel treatment options to stabilize renal function.

The pathogenesis of CKD is characterized by a progressive loss of kidney function, and the relentless accumulation and deposition of extracellular matrix (ECM), resulting in widespread renal fibrosis $(5,6)$. Histologically, the risk for ESRD and the rate of excretory function loss is positively correlated with the degree of tubular atrophy and interstitial fibrosis (7). Chronic tubulointerstitial hypoxia, one of numerous possible initial insults, has been shown to trigger tubulointerstitial pathology $(8,9)$. Previous research has demonstrated that hypoxia has a critical role in tubulointerstitial injury prior to microvasculature structural damage (8), suggesting pathogenic implications of hypoxia in the early stages of kidney disease. An important mechanism underlying the development of renal interstitial fibrosis is the epithelial-to-mesenchymal transition (EMT), a process in which the tubular epithelial cells lose their epithelial phenotype and acquire new phenotypical features, characteristic of mesenchymal cells, including the expression of mesenchymal markers and the acquisition of mobility (10). It has been observed, that under hypoxic conditions, myofibroblasts, which are transdifferentiated from tubular cells, are capable of migrating to the tubular interstitium, leading to renal fibrogenesis (11). Previous studies have 
demonstrated the importance of transcriptional responses to hypoxia, including the hypoxia-inducible factor (HIF-1), in mediating EMT $(12,13)$; however, the exact mechanisms underlying EMT induced by hypoxia remain largely unknown.

Adrenomedullin (ADM) is a vascular-derived peptide that was initially purified from a phaeochromocytoma (14) but has also been shown to be expressed in other body tissues, suggesting that it possesses numerous biological functions. In addition to being produced by both stromal (i.e., endothelial, vascular smooth muscle, myocardial and central nervous system) and tumor cells, as an autocrine/paracrine factor, ADM has been shown to be produced during the differentiation of macrophages in response to pro-inflammatory stimuli and hypoxia $(15,16)$. ADM is also implicated in renal fibrogenesis, and the expression of ADM and its receptor, in rat kidney interstitial cells, has been shown to inhibit cell proliferation and mRNA expression levels of ECM proteins (17). Nagae et al (18) showed that overexpression of exogenous ADM, in the ureteral-obstructed kidney, prevented tubulointerstitial fibrosis and cell proliferation. ADM signaling, however, has not yet been extensively explored during EMT, particularly with respect to its induction in response to hypoxia.

EMT is a process aggravated by chronic tubulointerstitial hypoxia, a condition that is believed to be associated with ADM production. Therefore, the aim of the present study was to verify whether ADM signaling was activated during EMT, and whether regulation of ADM could affect the development of EMT in human proximal tubular epithelial cells.

\section{Materials and methods}

Antibodies and reagents. A primary anti-ADM antibody was obtained from Abcam (Cambridge, MA, USA), and the anti-HIF-1 $\alpha$, anti-E-cadherin, anti-tight junction protein-1 (ZO-1), anti-vimentin and anti- $\alpha$-smooth muscle actin ( $\alpha$-SMA) antibodies were purchased from Santa Cruz Biotechnology Inc., (Dallas, TX, USA). The antibodies against total extracellular signal-regulated kinase (ERK), phospho-ERK (Thr202/Tyr204), and $\beta$-actin were purchased from Cell Signaling Technology Inc., (Danvers, MA, USA). Human ADM and ADM 22-52 (an ADM receptor antagonist), and apigenin (an activator of ERK) were obtained from Sigma-Aldrich (St. Louis, MO, USA).

Cell culture and treatment. The HK-2 and HKC human proximal tubular epithelial cells (American Type Culture Collection, Manassas, VA, USA) were cultured in Dulbecco's modified Eagle's medium/Ham's F12 (Invitrogen Life Technologies, Carlsbad, CA, USA), supplemented with $5 \mu \mathrm{g} / \mathrm{ml}$ insulin, $5 \mu \mathrm{g} / \mathrm{ml}$ transferrin, $5 \mathrm{ng} / \mathrm{ml}$ selenium, $36 \mathrm{ng} / \mathrm{ml}$ hydrocortisone, $4 \mathrm{pg} / \mathrm{ml}$ tri-iodo-L-thyronine, $10 \mathrm{ng} / \mathrm{ml}$ epidermal growth factor, $50 \mathrm{U} / \mathrm{ml}$ penicillin, $50 \mu \mathrm{g} / \mathrm{ml}$ streptomycin, and $2 \mathrm{mM}$ glutamine (all Invitrogen Life Technologies, Carlsbad, CA, USA). The HK-2 and HKC cells from passages 6-16 were used in the following experiments. The cells were starved of serum overnight, prior to experimentation, to avoid the effects of serum-induced signaling. The cells were maintained at $37^{\circ} \mathrm{C}$ in a humidified atmosphere, under normoxic $\left(21 \% \mathrm{O}_{2}\right)$ or hypoxic $\left(1 \% \mathrm{O}_{2}\right)$ conditions for 12,24 or $48 \mathrm{~h}$ with $95 \% \mathrm{~N}_{2}$ and $5 \% \mathrm{CO}_{2}$. In some experiments, the cells were exposed to normoxia or hypoxia in the absence or presence of the following compounds: 0.1 or $1.0 \mu \mathrm{M}$ ADM; 5 nM ADM22-52; and $100 \mu \mathrm{M}$ apigenin.

Gene silencing in the HK-2 cell line using ADM-small hairpin (sh)RNA. The HK-2 cells from the sixth passage were seeded in 6 -well plates $\left(1 \times 10^{5}\right.$ cells/well) and allowed to adhere for $24 \mathrm{~h}$, prior to transfection. The commercial ADM shRNA plasmid was purchased from Santa Cruz (Santa Cruz Biotechnology, Dallas, Texas, USA). The plasmid is based on a pool of 3 target-specific lentiviral vector plasmids, each encoding 19-25 nt (plus hairpin) shRNAs designed to knockdown human ADM gene expression. Each plasmid contains a puromycin resistance gene and a copGFP gene for the selection of cells stably expressing shRNA. The shRNAs were transfected using specifically designed reagent for shRNA transfection, including Santa Cruz Biotechnology's shRNA Plasmid Transfection Reagent (sc-108061, $0.2 \mathrm{ml}$ ) and shRNA Plasmid Transfection Medium (sc-108062, $20 \mathrm{ml}$ ) according to manufacturer's protocol. Then the cells were incubated for $8 \mathrm{~h}$ at $37^{\circ} \mathrm{C}$ in a $95 \%$ air $/ 5 \% \mathrm{CO}_{2}$ incubator. In addition, the cells were transfected with a non-targeting negative-control shRNA (NC-shRNA) (Santa Cruz Biotechnology, Dallas, Texas, USA) to monitor and optimize transfection efficiency. At $72 \mathrm{~h}$ post-transfection, puromycin $(7.0 \mu \mathrm{g} / \mathrm{ml})$ was added to the culture medium for selection and further characterization. The transfection efficiency of the HK-2 cells expressing ADM-shRNA (copGFP) was assessed using flow cytometry. In brief, transfected cell were fixed in cold $70 \%$ ethanol, washed twice and resuspended in cold phosphate-buffered saline buffer. The cell suspensions were analyzed on an FC500/MPL flow cytometer (Beckman Coulter, Brea, CA), using the FlowJo software (Tree Star, Ashland, OR, USA). GFP was detected on the FITC channel using a $488 \mathrm{~nm}$ laser, and the transfection efficiency was no less than $85 \%$.. The cells were cultured for $48 \mathrm{~h}$ following transfection and then exposed to hypoxia for $12-48 \mathrm{~h}$.

Cell lysis and western blot analysis. The treated HK-2 and $\mathrm{HKC}$ cells were harvested and lysed for $30 \mathrm{~min}$ on ice using lysis buffer (50 mM Tris-HCl, pH 7.4; 1\% Nonidet P-40; $0.25 \%$ sodium deoxycholate; $150 \mathrm{mM} \mathrm{NaCl} ; 1 \mathrm{mM}$ EDTA; $1 \mathrm{mM}$ PMSF; $1 \mu \mathrm{g} / \mathrm{ml}$ each aprotinin, leupetin, and pepstatin; $1 \mathrm{mM} \mathrm{Na} \mathrm{VO}_{4} ; 1 \mathrm{mM} \mathrm{NaF}$ ). The samples were then centrifuged at $14,000 \times \mathrm{g}$ for $10 \mathrm{~min}$ at $4^{\circ} \mathrm{C}$, and the protein concentrations of the cell lysates were determined using the Bradford method. The proteins (50 $\mu \mathrm{g} / \mathrm{lane}$ ) were loaded onto $10 \%$ SDS-containing polyacrylamide gels and were separated by SDS-PAGE, prior to a transfer to polyvinylidene fluoride membranes (Millipore, Bedford, MA, USA). The membranes were blocked with $5 \%$ milk in Tris-buffered saline (TBS) with Tween ${ }^{\circledR} 20(20 \mathrm{mM}$ Tris- $\mathrm{HCl}, 150 \mathrm{mM} \mathrm{NaCl}$, and $0.1 \%$ Tween $^{\circledR} 20$ ) for $1 \mathrm{~h}$ at room temperature. Subsequently, the membranes were incubated overnight at $4^{\circ} \mathrm{C}$ with the primary antibodies, in blocking buffer containing $5 \%$ milk, at the dilutions specified in the manufacturer's instructions. Following extensive washing in TBS, the membranes were incubated with the appropriate horseradish peroxidase-conjugated secondary antibodies (Santa Cruz, Dallas, Texas, USA), for $1 \mathrm{~h}$ at room temperature in $5 \%$ nonfat milk dissolved in 
TBS. The membranes were visualized using a chemiluminescent substrate and developed following exposure to Kodak X-OMAT film (Kodak, Rochester, NY, USA). The immunoreactive protein bands were quantified by densitometry using QuantityOne Image Analysis Software (Bio-Rad Laboratories, Hercules, CA, USA) and were normalized to the $\beta$-actin values.

Quantitative polymerase chain reaction ( $q P C R$ ). Total RNA was extracted from treated cells using the RNAiso Plus kit (Takara Biotechnology Co., Ltd., Dalian, China). A total of $1 \mu \mathrm{g}$ RNA was used for first-strand cDNA synthesis using the PrimeScript reverse trasncription kit reagents (Takara Biotechnology Co., Ltd.). Quantification was performed using the $\mathrm{iCycler}_{\mathrm{iQ}}{ }^{\mathrm{TM}}$ Sequence Detection system (Bio-Rad, USA), and the SYBR Green Premix kit (Takara Biotechnology Co., Ltd.) was used as the fluorophore. The following oligonucleotides were used as primers: ADM forward, 5'-ACTTGGCAGATCACTCTCTTAGCA-3', and reverse, 5'-ATCAGGGCGACGGAAACC-3'; $\beta$-actin forward, 5'-ATCCTGCCAGTAGCATATGC-3', and reverse, 5'-ACCGGGTTGGTTTTGATCTG-3'. For each primer pair, the annealing temperature was optimized using a gradient PCR reaction. The expression levels of each target mRNA, relative to the $\beta$-actin mRNA expression levels, were calculated from the cycle threshold $(\mathrm{Ct})$ using the $2^{-\Delta \Delta \mathrm{Ct}}$ formula. For each sample, the experiment was repeated in triplicate, using RNA from three separate culture wells.

Statistical analysis. All experimental data are expressed as the means \pm standard error of the mean. The significant differences were evaluated using a one- or two-way analysis of variance, followed by multiple comparisons tests. Statistical analyses were performed using the commercially available software package SPSS version 13 (SPSS, Inc., Chicago, IL, USA). A $\mathrm{P}<0.05$ was considered to indicate a statistically significant difference.

\section{Results}

Hypoxia induces EMT in human tubular epithelial cells and increases the expression of ADM at the transcriptional level. The HK-2 and HKC cells, cultured under chronic hypoxia for $48 \mathrm{~h}$, exhibited a downregulation in the expression levels of the epithelial markers, E-cadherin and ZO-1, and an upregulation in the expression levels of the mesenchymal markers, vimentin and $\alpha$-SMA, as compared with the cells cultured under normoxia (Fig. 1). The expression levels of the hypoxia-regulated molecule HIF-1 $\alpha$ were also determined to confirm the hypoxic response. These results suggest that the epithelial cells underwent EMT, with a more fibroblast-like cell type, and indicate that hypoxia may promote EMT, one of the major mechanisms that mediates renal fibrosis. Furthermore, ADM expression levels were shown to be increased in $\mathrm{HKC}$ and HK-2 cells under hypoxic conditions, and was negatively correlated to the expression levels of E-cadherin and ZO-1.

ADM mRNA expression was evaluated in the HK-2 and HKC cells, by qPCR. The mRNA expression of $\beta$-actin was used as an internal control, and the ratio of $\mathrm{ADM} / \beta$-actin was used to determine the relative mRNA expression levels of ADM. As shown in Fig. 2, the relative expression levels of ADM mRNA, in HKC and HK-2 cells, were significantly affected by both the time the cells were exposed to hypoxia $\left(\mathrm{F}_{\text {time }(3,24)}=52.43, \mathrm{P}<0.0001\right)$ and the type of cell line $\left(\mathrm{F}_{\text {cell lines }(1,24)}=30.90, \mathrm{P}<0.0001\right)$; these two factors also showed significant interactions $\left(\mathrm{F}_{\text {time } \mathrm{x} \text { cell lines }(3,24)}=8.57, \mathrm{P}<0.0005\right)$. A post hoc analysis showed that the mRNA expression levels of ADM significantly increased in both HKC and HK-2 cells, as a function of the level of hypoxia. Between the two cell lines, the HK-2 cells appeared more sensitive to hypoxic stress, as compared with the HKC cells $(\mathrm{P}<0.0001$, at $48 \mathrm{~h}$ of hypoxia). These results suggest that expression of ADM was significantly enhanced in a time-dependent manner in human tubular cell lines under hypoxic conditions.

Hypoxia-induced EMT is prevented by exogenous administration of $A D M$. ADM was shown to be upregulated in hypoxia-stimulated $\mathrm{HK}-2$ and $\mathrm{HKC}$ cells, therefore it was assessed as to whether exogenous ADM was capable of ameliorating hypoxia-induced EMT. HK-2 cells were exposed to hypoxia for $48 \mathrm{~h}$ in the presence of $0,0.1$ or $1.0 \mu \mathrm{M}$ ADM. As shown in Fig. 3, the expression levels of E-cadherin were significantly increased in response to $1.0 \mu \mathrm{M}$ ADM, as compared with the control cells not treated with ADM $(\mathrm{P}<0.0001)$; however, this upregulation did not occur when the ADM peptide inhibitor ADM 22-52 was co-administered with $1.0 \mu \mathrm{M}$ ADM. Furthermore, the protein expression levels of vimentin were significantly decreased by ADM, in a dose-dependent manner $(\mathrm{P}<0.05$ and $\mathrm{P}<0.0001$, respectively); however, this downregulation did not occur when ADM was co-administered with ADM 22-52. These results suggest that exogenous ADM administration ameliorated hypoxia-induced EMT.

ADM knockdown accelerates EMT in human tubular epithelial cells. To further elucidate the effects of ADM on hypoxia-induced EMT in human tubular epithelial cells, a stable cell line was constructed, in which ADM expression was successfully knocked down. Because HK-2 cells appeared to have increased sensitivity to hypoxic stress, lentiviral ADM-shRNA was transfected into the HK-2 cells. Following the transfection, western blot analysis revealed that ADM protein expression levels were markedly decreased in the HK-2 cells, as compared with the parental cells (Fig. 4A, $\mathrm{P}<0.01$ ). The expression levels of ADM were not different between the vector-control and untransfected parental HK-2 cells. Following $48 \mathrm{~h}$ exposure to hypoxia, the HK-2 cells that stably expressed ADM-shRNA exhibited significantly lower expression levels of E-cadherin, as compared with the untransfected cells (Fig. 4B, P<0.05). There was no difference in the vimentin protein expression levels between the parental and vector-control cells, under hypoxic conditions; however, vimentin expression levels were markedly upregulated in the hypoxic shRNA-ADM-transfected cells $(\mathrm{P}<0.05)$. These results indicate that ADM knockdown may accelerate EMT in tubular epithelial cells, and that this effect may be partly mediated by hypoxia-induced EMT processes.

Effects of hypoxia-induced ADM on ameliorating EMT are regulated by the inhibition of the activation of ERK. It was investigated whether the upregulated expression of ADM ameliorated hypoxia-induced EMT, through inhibiting the 

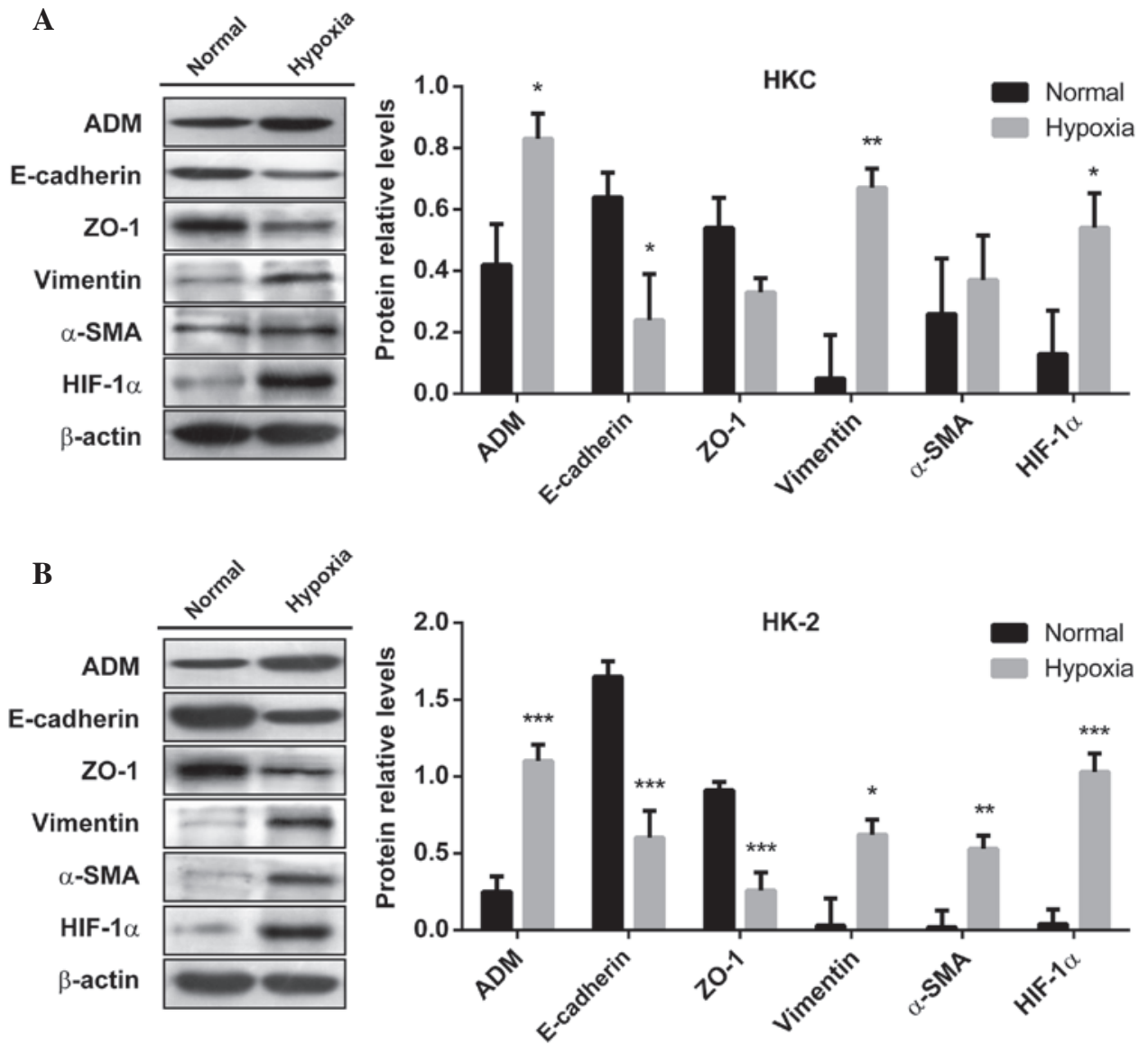

Figure 1. Hypoxia induces epithelial-to-mesenchymal transition in HKC and HK-2 human proximal tubular epithelial cells. (A) HKC and (B) HK-2 cells were cultured under hypoxic conditions for $48 \mathrm{~h}$, and the expression of adrenomedullin (ADM), E-cadherin, tight junction protein-1 (ZO-1), vimentin, $\alpha$-smooth muscle actin $(\alpha-\mathrm{SMA})$ and hypoxia inducible factor (HIF)-1 $\alpha$ were analyzed by western blotting. The data represent the means \pm standard error of the mean. ${ }^{*} \mathrm{P}<0.05,{ }^{* *} \mathrm{P}<0.01,{ }^{* * *} \mathrm{P}<0.0001$, as compared with the cells cultured in normoxic conditions.

activation of ERK. The HK-2 cells were exposed to hypoxia for $48 \mathrm{~h}$ in the presence of $1.0 \mu \mathrm{M} \mathrm{ADM}$, with or without apigenin $(100 \mu \mathrm{M})$. As shown in Fig. 5, the phosphorylation of ERK was significantly decreased in the ADM-treated cells, as compared with the control cells $(\mathrm{P}<0.01)$. However, when the HK-2 cells were treated with exogenous ADM in combination with apigenin, the levels of phospho-ERK were significantly elevated $(\mathrm{P}<0.05)$. Conversely, total ERK expression levels were not affected by either ADM nor apigenin treatment $\left(\mathrm{F}_{(2,15)}=0.1833, \mathrm{P}=0.83\right)$. E-cadherin expression levels were significantly increased by $1.0 \mu \mathrm{M}$ ADM $(\mathrm{P}<0.0001)$, as compared with the control cells not treated with ADM; however, $100 \mu \mathrm{M}$ apigenin blocked the ADM-induced upregulation of E-cadherin. The expression levels of vimentin were significantly decreased by ADM $(\mathrm{P}<0.01)$; however, this downregulation was completely prevented by treatment with ADM in combination with apigenin $(\mathrm{P}<0.0001)$. These results suggest that the effects of hypoxia-induced ADM on the amelioration of EMT may be regulated by inhibition of the activation of ERK, and this effect can be effectively prevented by the administration of activators of ERK.

\section{Discussion}

It has previously been reported that a hypoxic environment is associated with the induction of EMT in renal

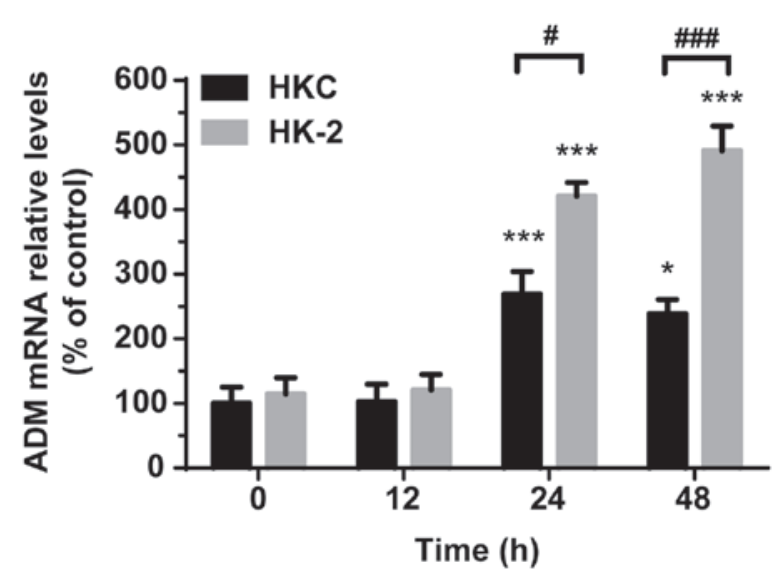

Figure 2. Hypoxia increases the expression of adrenomedullin (ADM) at the transcriptional level. HKC and HK-2 human proximal tubular epithelial cells were cultured under hypoxic conditions for $0,12,24$ and $48 \mathrm{~h}$. The ADM mRNA expression levels were analyzed by quantitative polymerase chain reaction. The data represent the means \pm standard error of the mean. ${ }^{*} \mathrm{P}<0.05,{ }^{* * *} \mathrm{P}<0.0001$, as compared with $0 \mathrm{~h}$ (equivalent to normoxic conditions); ${ }^{\#} \mathrm{P}<0.05,{ }^{\# \#} \mathrm{P}<0.0001$, as compared with the indicated group. H, hours.

fibrogenesis (19). A previous study demonstrated that overexpression of exogenous ADM in the ureteral-obstructed kidney prevented tubulointerstitial fibrosis and cell proliferation (18). In the present study, ADM signaling was shown to 


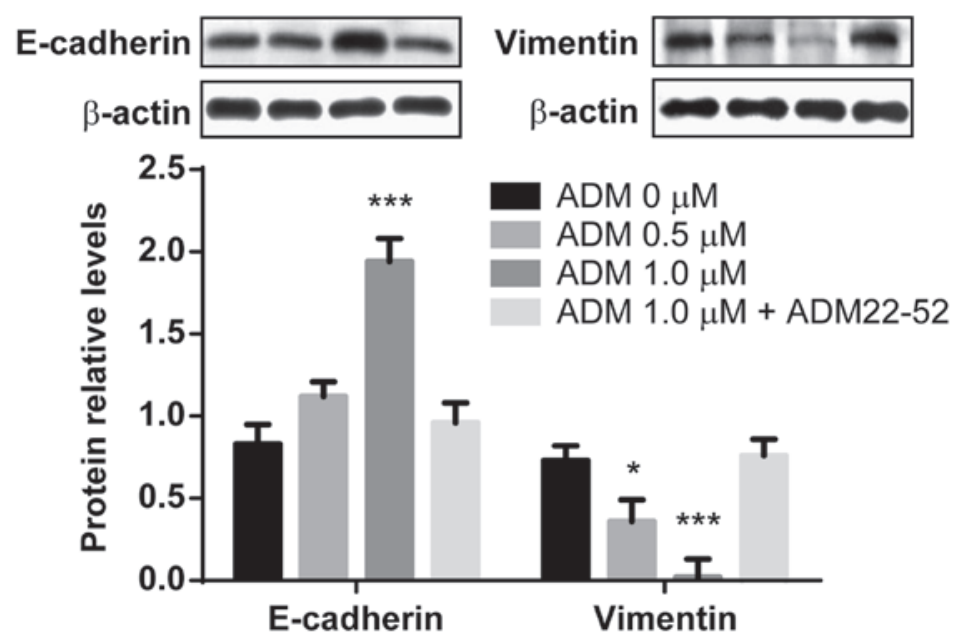

Figure 3. Hypoxia-induced epithelial-to-mesenchymal transition is prevented by exogenous adrenomedullin (ADM) administration. HK-2 human proximal tubular epithelial cells were exposed to hypoxia for $48 \mathrm{~h}$ in the presence of 0, 0.1, 1.0 $\mu \mathrm{M}$ ADM or co-administered with ADM peptide inhibitor ADM 22-52. The protein expression levels of E-cadherin and vimentin were determined by western blot analysis. The data represent the means \pm standard error of the mean. ${ }^{*} \mathrm{P}<0.05,{ }^{* * *} \mathrm{P}<0.0001$, as compared with the controls.
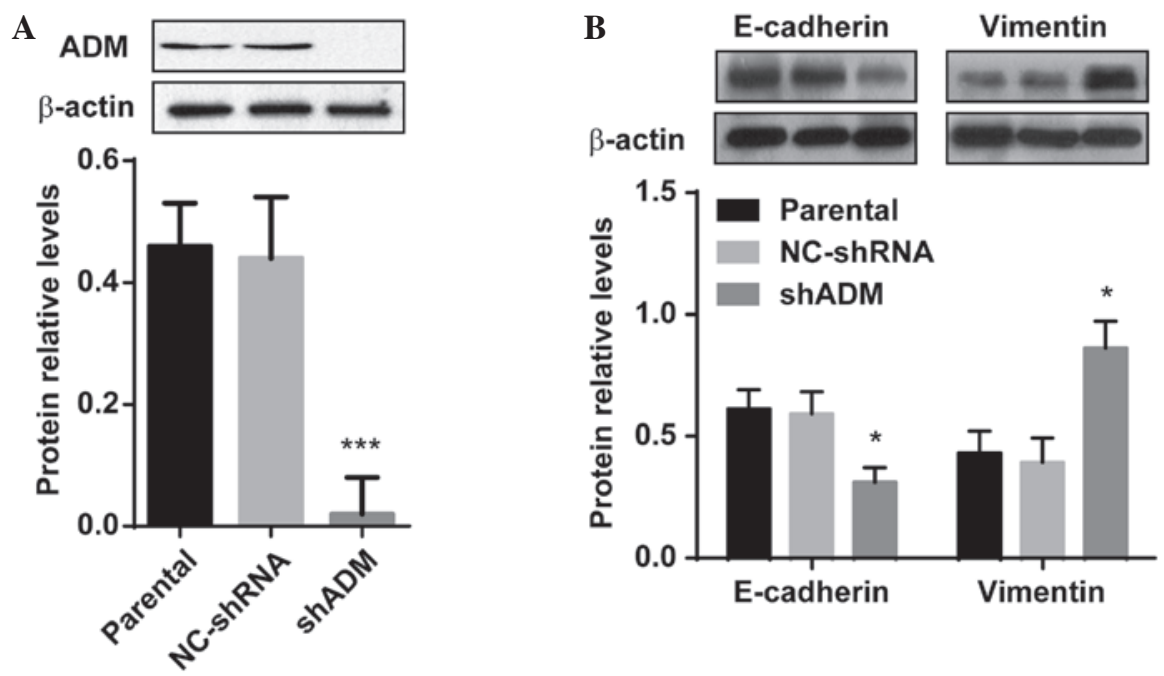

Figure 4. Knockdown of adrenomedullin (ADM) expression accelerated the epithelial-to-mesenchymal transition in HK-2 human proximal tubular epithelial cells. ADM was knocked down in HK-2 cells by lentiviral ADM-small hairpin (sh)RNA transfection. (A) Following the transfection, the protein expression levels of ADM were determined. (B) Following $48 \mathrm{~h}$ of culturing in hypoxic conditions, the protein expression levels of E-cadherin and vimentin were also analyzed in HK-2 cells that stably expressed ADM-shRNA. The data represent the means \pm standard error of the mean. $\mathrm{P}<0.05,{ }^{* * *} \mathrm{P}<0.0001$, as compared with the controls (NC-shRNA) and parental cells.
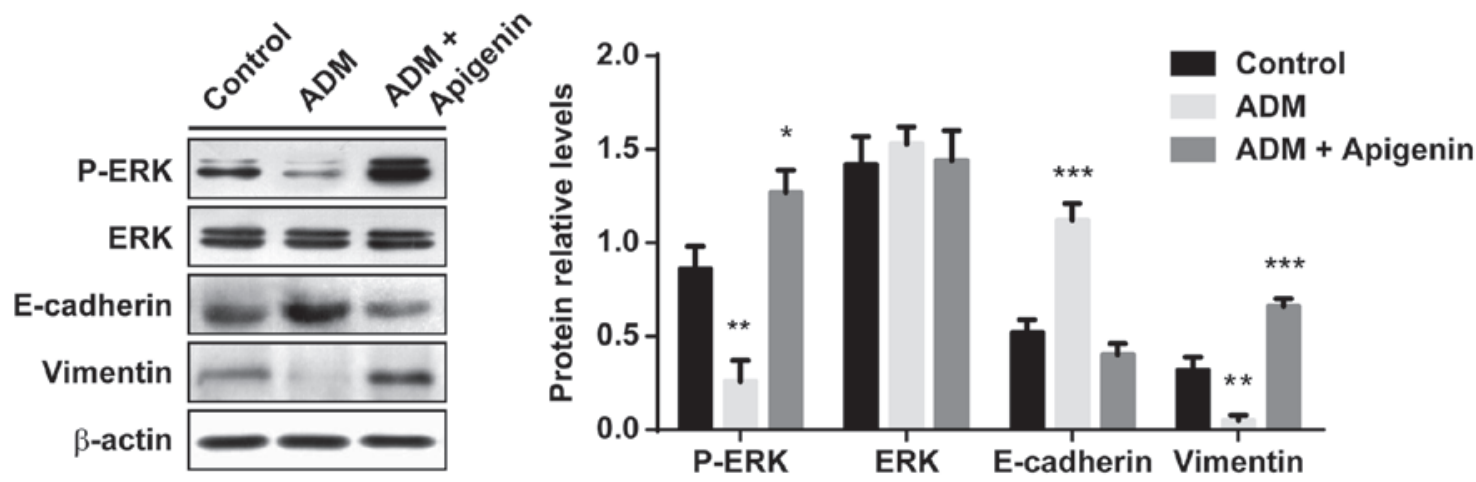

Figure 5. The effects of hypoxia-induced adrenomedullin (ADM) on ameliorating epithelial-to-mesenchymal transition is regulated by inhibition of the activation of extracellular signal-regulated kinase (ERK). HK-2 human proximal tubular epithelial cells were exposed to hypoxia for $48 \mathrm{~h}$ in the presence of $1.0 \mu \mathrm{M}$ ADM, with or without apigenin $(100 \mu \mathrm{M})$. The phosphorylation of ERK (P-ERK) and expression levels of ERK, E-cadherin and vimentin were analyzed by western blotting. The data represent the means \pm standard error of the mean. ${ }^{*} \mathrm{P}<0.05,{ }^{* * *} \mathrm{P}<0.01,{ }^{* * * * *} \mathrm{P}<0.0001$, as compared with the controls. 
be active in human tubular epithelial cells, under hypoxic conditions. Overexpression of ADM significantly increased the expression levels of the epithelial markers and decreased the expression levels of the mesenchymal markers; whereas the knockdown of ADM expression suppressed the epithelial phenotype. ADM protected the HK-2 and HKC cells from EMT, via the inhibition of the activation of ERK, and the ADM-mediated protection was prevented by administration of an ADM peptide inhibitor. These data provide an explanation for the protective effects of ADM on renal fibrogenesis, through the suppression of EMT.

Chronic hypoxia is a principal contributing factor associated with end-stage renal failure. Previous experimental evidence has shown that the proximal tubular epithelial cells adapt to hypoxic environments through the transcriptional activation of HIF-1 (20). This activation leads to alterations in the composition of the secreted proteins, which are released primarily into the basolateral compartment of the kidney. These secreted proteins favor the recruitment of inflammatory cells into the renal interstitium and contribute to fibrogenic reactions (21). Furthermore, it has been suggested that in certain circumstances, tubular expression of antifibrotic cytokines protects the proximal tubular epithelial cells from apoptosis, and reduces the interstitial collagen accumulation (22). The present study demonstrated the hypoxia-mediated induction of ADM expression in human tubular epithelial cells.

It has previously been observed that hypoxia can increase the expression levels of both ADM mRNA and secreted protein, in various of tissues $(23,24)$. These increases are at least partly mediated by the activation of HIF-1 (25). Gene expression profiling and a systems biology analysis previously identified the activation of intracellular vascular endothelial growth factor signaling and hypoxia-response pathways in patients with progressive proteinuric glomerulopathiesl; these pathways are associated with the upregulation of HIF-1 $\alpha$ and numerous HIF target genes (26). In addition, the expression of ADM and its receptor has been shown in renal tubular cell lines (27). The results of the present study suggested that ADM expression is higher in human proximal tubular epithelial cells under hypoxia, as compared with the cells cultured under normoxia, and the increased expression of ADM may protect the kidney against ischemic injury. Shah et al (28) previously reported that ADM, combined with ADM binding protein-1, can prevent and/or minimize damage in a rat model of renal ischemia and reperfusion injury.

Previous studies have shown that EMT, a process by which differentiated epithelial cells undergo a phenotypic conversion that results in the production of matrix-producing fibroblasts and myofibroblasts, has a critical role in the development of renal interstitial fibrosis. Chronic hypoxia may affect the development of renal fibrogenesis in CKD, through EMT. The morphology of tubular epithelial cells and interstitial myofibroblasts are markedly different, and the phenotypically altered cells are located in tissue compartments that are separated by the tubular basement membranes within the kidney. Gene expression profiling, using microarray technology, has identified numerous genes with altered expression patterns during tubular EMT $(29,30)$. Previously, Sun et al (13) showed that under hypoxic conditions, HIF-1a induced the overexpression of Twist, a basic helix-loop-helix transcription factor, that lead to the promotion of EMT in human tubular epithelial cells. The results of the present study suggested that overexpression of ADM protected tubular epithelial cells from converting to interstitial fibroblasts, as indicated by ADM-induced increased expression levels of the epithelial markers (E-cadherin and ZO-1) and decreased expression levels of the mesenchymal markers vimentin and $\alpha$-SMA, during hypoxia. E-cadherin is associated with the actin filament network through catenins, a family of intracellular adhesive junction proteins (31). The importance of E-cadherin in the development of normal epithelium, has been established using knockout mice, in which its role in embryonic epitheliogenesis during kidney development was demonstrated (32). Furthermore, the expression levels of ZO-1, a component of the tight junctions between epithelial cells, were found to be suppressed during EMT in previous studies $(33,34)$. Such alterations may consequently result in the destabilization of the structural integrity of the renal epithelium, and induce the cells to lose their epithelial adhesion properties and polarity. The reorganization of the actin cytoskeleton and the induction of $\alpha$-SMA may provide a structural foundation, not only for defining the morphology of the transformed cells, but also to explain their ability to migrate, invade, and acquire the capacity for contractility. In addition to the actin cytoskeleton, cytoplasmic intermediate filaments also undergo conversion from an epithelial type of cytokeratin to the mesenchymal vimentin (34-36).

Tubular epithelial cells can acquire a mesenchymal phenotype, through the process of EMT. This allows for an enhanced migratory capacity, enabling the cells to shift from the renal tubular microenvironment, into the interstitium and avoid undergoing apoptosis. Previous findings have suggested that EMT may be reversible in tubular epithelial cells; however, this is dependent on the surviving cells to repopulate the injured tubules with functional epithelia (37). The major regulators of renal epithelial cell plasticity are bone morphogenic protein-7 (BMP-7) and transforming growth factor (TGF)- $\beta 1$. TGF- $\beta 1$ is known to be associated with the induction of EMT in renal tubular epithelial cells (10), whereas BMP-7 has been shown to oppose TGF- $\beta 1$-induced EMT and reverse chronic renal injury (33). Previous studies have suggested that numerous mitogenic signaling components; such as, protein kinase $\mathrm{C}$, mitogen activated protein kinase kinase and oxidant, are associated with the regulation of TGF- $\beta 1$ (38), and that activation of ERK is involved in renal fibrosis following kidney damage (39). In the present study, ERK activity was shown to be markedly inhibited, following exogenous administration of ADM, in proximal tubular epithelial cells. The effects of ADM on EMT were effectively prevented when an activator of ERK, apigenin, was co-administered with ADM. These observations imply that ADM may suppress EMT through the inhibition of ERK signaling in human tubular epithelial cells.

In conclusion, the present study demonstrated that ADM signaling is induced by hypoxia and is functionally active in human proximal tubular epithelial cells. Furthermore, ADM was shown to inhibit hypoxia-induced EMT, which has a critical role in the promotion of renal fibrogenesis. Therefore, the selective upregulation of ADM signaling may be therapeutically efficacious in patients with CKD. 


\section{References}

1. Klahr S and Morrissey J: Progression of chronic renal disease. Am J Kidney Dis 41: S3-S7, 2003.

2. Owen WF Jr: Patterns of care for patients with chronic kidney disease in the United States: dying for improvement. J Am Soc Nephrol 14: S76-S80, 2003

3. Coresh J, Selvin E, Stevens LA, et al: Prevalence of chronic kidney disease in the United States. JAMA 298: 2038-2047, 2007

4. Centers for Disease Control and Prevention: Prevalence of chronic kidney disease and associated risk factors - United States, 1999-2004. MMWR Morb Mortal Wkly Rep 56: 161-165, 2007.

5. Liu Y: Renal fibrosis: new insights into the pathogenesis and therapeutics. Kidney Int 69: 213-217, 2006.

6. Strutz F and Zeisberg M: Renal fibroblasts and myofibroblasts in chronic kidney disease. J Am Soc Nephrol 17: 2992-2998, 2006.

7. Nath KA: Tubulointerstitial changes as a major determinant in the progression of renal damage. Am J Kidney Dis 20: 1-17, 1992

8. Nangaku M: Chronic hypoxia and tubulointerstitial injury: a final common pathway to end-stage renal failure. J Am Soc Nephrol 17: 17-25, 2006.

9. Nangaku M and Eckardt KU: Hypoxia and the HIF system in kidney disease. J Mol Med (Berl) 85: 1325-1330, 2007.

10. Zeisberg M and Kalluri R: The role of epithelial-to-mesenchymal transition in renal fibrosis. J Mol Med (Berl) 82: 175-181, 2004.

11. Manotham K, Tanaka T, Matsumoto M, et al: Transdifferentiation of cultured tubular cells induced by hypoxia. Kidney Int 65: 871-880, 2004

12. Higgins DF, Kimura K, Bernhardt WM, et al: Hypoxia promotes fibrogenesis in vivo via HIF-1 stimulation of epithelial-to-mesenchymal transition. J Clin Invest 117: 3810-3820, 2007.

13. Sun S, Ning X, Zhang Y, et al: Hypoxia-inducible factor-1alpha induces Twist expression in tubular epithelial cells subjected to hypoxia, leading to epithelial-to-mesenchymal transition. Kidney Int 75: 1278-1287, 2009.

14. Kitamura K, Kangawa K, Kawamoto M, et al: Adrenomedullin: a novel hypotensive peptide isolated from human pheochromocytoma. Biochem Biophys Res Commun 192: 553-560, 1993.

15. Hinson JP, Kapas S and Smith DM: Adrenomedullin, a multifunctional regulatory peptide. Endocr Rev 21: 138-167, 2000

16. Chen L, Qiu JH, Zhang LL and Luo XD: Adrenomedullin promotes human endothelial cell proliferation via HIF-1 $\alpha$. Mol Cell Biochem 365: 263-273, 2012.

17. Eto Y, Shimosawa T, Nitta K, Nihei H and Maruyama N: Interaction between adrenomedullin and angiotensin II in DNA synthesis and extracellular matrix accumulation in cultured rat kidney interstitial cells. Clin Exp Nephrol 6: 7-12, 2002.

18. Nagae T, Mori K, Mukoyama M, et al: Adrenomedullin inhibits connective tissue growth factor expression, extracellular signal-regulated kinase activation and renal fibrosis. Kidney Int 74: 70-80, 2008.

19. Norman JT, Clark IM and Garcia PL: Hypoxia promotes fibrogenesis in human renal fibroblasts. Kidney Int 58: 2351-2366, 2000

20. Leonard MO, Cottell DC, Godson C, Brady HR and Taylor CT: The role of HIF-1 alpha in transcriptional regulation of the proximal tubular epithelial cell response to hypoxia. J Biol Chem 278: 40296-40304, 2003.

21. Rudnicki M, Eder S, Perco P, et al: Gene expression profiles of human proximal tubular epithelial cells in proteinuric nephropathies. Kidney Int 71: 325-335, 2007.
22. Wang S, de Caestecker M, Kopp J, Mitu G, Lapage J and Hirschberg R: Renal bone morphogenetic protein-7 protects against diabetic nephropathy. J Am Soc Nephrol 17: 2504-2512, 2006.

23. Ogita T, Hashimoto E, Yamasaki M, et al: Hypoxic induction of adrenomedullin in cultured human umbilical vein endothelial cells. J Hypertens 19: 603-608, 2001.

24. Oehler MK, Norbury C, Hague S, Rees MC and Bicknell R: Adrenomedullin inhibits hypoxic cell death by upregulation of Bcl-2 in endometrial cancer cells: a possible promotion mechanism for tumour growth. Oncogene 20: 2937-2945, 2001.

25. Nguyen SV and Claycomb WC: Hypoxia regulates the expression of the adrenomedullin and HIF-1 genes in cultured HL-1 cardiomyocytes. Biochem Biophys Res Commun 265: 382-386, 1999.

26. Rudnicki M, Perco P, Enrich J, et al: Hypoxia response and VEGF-A expression in human proximal tubular epithelial cells in stable and progressive renal disease. Lab Invest 89: 337-346, 2009.

27. Sato K, Imai T, Iwashina M, Marumo F and Hirata Y: Secretion of adrenomedullin by renal tubular cell lines. Nephron 78: 9-14, 1998.

28. Shah KG, Rajan D, Jacob A, et al: Attenuation of renal ischemia and reperfusion injury by human adrenomedullin and its binding protein. J Surg Res 163: 110-117, 2010.

29. Zavadil J, Bitzer M, Liang D, et al: Genetic programs of epithelial cell plasticity directed by transforming growth factor-beta. Proc Natl Acad Sci USA 98: 6686-6691, 2001.

30. Jechlinger M, Grunert S, Tamir IH, et al: Expression profiling of epithelial plasticity in tumor progression. Oncogene 22: 7155-7169, 2003.

31. Bajpai S, Feng Y, Wirtz D and Longmore GD: $\beta$-Catenin serves as a clutch between low and high intercellular E-cadherin bond strengths. Biophys J. 105: 2289-2300, 2013

32. Horster MF, Braun GS and Huber SM: Embryonic renal epithelia: induction, nephrogenesis, and cell differentiation. Physiol Rev 79: 1157-1191, 1999.

33. Zeisberg M, Hanai J, Sugimoto $\mathrm{H}$, et al: BMP-7 counteracts TGF-beta1-induced epithelial-to-mesenchymal transition and reverses chronic renal injury. Nat Med 9: 964-968, 2003.

34. OkadaH,Danoff TM,Kalluri R and Neilson EG: Early role of Fsp1 in epithelial-mesenchymal transformation. Am J Physiol 273: F563-F574, 1997.

35. Yang J and Liu Y: Dissection of key events in tubular epithelial to myofibroblast transition and its implications in renal interstitial fibrosis. Am J Pathol 159: 1465-1475, 2001.

36. Strutz F, Zeisberg M, Ziyadeh FN, et al: Role of basic fibroblast growth factor- 2 in epithelial-mesenchymal transformation. Kidney Int 61: 1714-1728, 2002.

37. Keller C, Kroening S, Zuehlke J, Kunath F, Krueger B and Goppelt-Struebe M: Distinct mesenchymal alterations in $\mathrm{N}$-cadherin and E-cadherin positive primary renal epithelial cells. PLoS One 7(8):e43584, 2012.

38. Grewal JS, Mukhin YV, Garnovskaya MN, Raymond JR and Greene EL: Serotonin 5-HT2A receptor induces TGF-beta1 expression in mesangial cells via ERK: proliferative and fibrotic signals. Am J Physiol 276: F922-F930, 1999.

39. Pat B, Yang T, Kong C, Watters D, Johnson DW and Gobe G: Activation of ERK in renal fibrosis after unilateral ureteral obstruction: modulation by antioxidants. Kidney In 67: 931-943, 2005. 